\title{
The Impact of COVID-19 on Cancer Risk and Treatment
}

\author{
Nidhi Jyotsana (D) and Michael R. King \\ Department of Biomedical Engineering, Vanderbilt University, Nashville, TN, USA
}

(Received 29 May 2020; accepted 18 June 2020; published online 29 June 2020)

Associate Editor Owen McCarty oversaw the review of this article.

\begin{abstract}
Millions of people are being infected with COVID-19 around the globe. Though the majority of them will recover, cancer patients remain at a higher risk to SARSCoV-2 infection and its related severe outcomes. Understanding how viruses contribute to human cancers provides us with new opportunities for preventing or treating virusassociated cancers. However, a limited amount of research has been done to date in the context of how viral infections impact cancer at the cellular level and vice versa. Therefore, in light of the COVID-19 global infection, this review highlights the need for better understanding of the biology of viral infections in cancer patients, to enable novel therapies to co-target viral infections and cancer.
\end{abstract}

Keywords-COVID-19, SARS-CoV-2, Cancer, Immunotherapy, Cytokine storm, ACE2, Coagulopathy.

\section{INTRODUCTION}

For the past three decades, viral infections have presented a great challenge for cancer patients and oncologists. COVID-19 (coronavirus disease of 2019), caused by the SARS-CoV-2 (Severe Acute Respiratory Syndrome-Coronavirus-2) virus, continues to spread around the globe (infectivity index, R $0 \sim 2.5$ ). The mortality rate of cancer patients who contracted SARS-CoV-2 virus was reported to be $6 \%$ in comparison to $1 \%$ for healthy people in China. ${ }^{22,59}$ Cancer patients are reported three times more susceptible to SARS-CoV-2 infection with possible poor prognosis than individuals without cancer because of their systemic immunosuppressive state caused by the malignancy and anticancer treatments, such as chemotherapy or surgery ( $A A C R$ Virtual Meeting, 2020). The continuously increasing numbers of cancer

Address correspondence to Nidhi Jyotsana, Department of Biomedical Engineering, Vanderbilt University, Nashville, TN, USA. Electronic mail: nidhi.jyotsana@vanderbilt.edu patients and the increase in numbers of COVID-19 infections in cancer patients either undiagnosed, diagnosed, under treatment or under remission, have provided a sense of urgency to understand the interconnection and develop novel therapies to cotarget viral infections and cancer. However, little is known about novel SARS-CoV-2 biology and very limited to no research has been done in the context of whether and how SARS-CoV-2 infection impacts cancer cells. In this review, we provide a brief overview of the impact that COVID-19 has in cancer emergence and treatment, and highlight the emerging need to study the role of COVID-19 infection in cancer progression and treatment.

\section{VIRAL INFECTIONS AND CANCER}

The inability of the immune system to distinguish between self and non-self, results in, and links, the pathogenesis of cancer and viral infections. Both viruses and cancers express proteins that are recognizable by host $\mathrm{T}$ cells and both prompt $\mathrm{T}$ cell mediated inflammation. ${ }^{23} \mathrm{~A}$ number of oncogenic (HBV, HCV, HPV, EBV, HIV) and oncolytic viruses (Coxsackievirus, reovirus, vaccinia virus, adenovirus) are known to cause and regress various cancer types, respectively. There is a 3rd category of non-oncogenic, non-oncolytic viruses that remain understudied for their contribution in cancer growth or regression. Conflicting results for the link between cancer subtypes and viral infections have been reported in clinical and pre-clinical studies. ${ }^{9,30,40}$ For instance, a faster growth of melanoma was observed in mice challenged with lymphocytic choriomeningitis virus and $\mathrm{PR} 8 / \mathrm{H} 1 \mathrm{~N} 1 /$ influenza A, respectively compared to the uninfected controls. ${ }^{29,30}$ This was attributed to the shunting of anti-cancer cytotoxic $\mathrm{CD} 8+\mathrm{T}$ cells from tumor site to the viral infection site. ${ }^{30}$ On the contrary, a slower 
growth of Lewis lung carcinoma cells was observed in mice that were infected with influenza virus. ${ }^{25}$ Infection with Drosophila C virus caused an almost 30\% reduction in wing discs and wing tumorigenesis in Drosophila through unknown mechanisms. ${ }^{43}$ The risk of Herpes Zoster infection in cancer patients compared to the general population is 2 to 8 -fold higher. ${ }^{20,24}$ With HIV-associated immune activation on the one hand and immune senescence on the other hand, the comorbidity of HIV and cancer remains complex and multifactorial. ${ }^{46}$ Viral infection-mediated chronic inflammation has long been associated with increased tumor growth and metastasis, and non-steroidal antiinflammatory drugs have been shown to reduce tumor growth and cancer development. ${ }^{10,57}$ It was reported that lung cancer patients showed lower immune response to influenza infection due to lower levels of interferon-beta compared to healthy controls. These lower interferon-beta levels were associated with the presence of tumor-derived exosomes that transfer epidermal growth factor receptor (EGFR) protein to immune cells such as macrophages. These macrophages could not produce sufficient interferon-beta in response to viral infection. ${ }^{17}$ This suggests that the course and outcome of the two diseases are defined by factors including the type of viral infection, type of cancer, and the immune system components involved.

\section{COVID-19 AND CANCER}

Coronaviruses are known to primarily target the human respiratory system. COVID-19 represents the seventh member of the coronavirus family that infects humans. COVID-19 by itself remains biologically novel and not much is known about the role of novel coronavirus SARS-CoV-2 in cancer. Similar to other severe acute respiratory outbreaks (SARS-CoV, MERS-CoV), comorbidities such as hypertension and malignancy predispose COVID-19-positive patients to adverse clinical outcomes. ${ }^{1,19,36,37}$ A recent study including 1590 COVID-19 positive patients in China shows cancer as one of the more serious comorbidities that increase risk with respect to COVID-19. ${ }^{19,22}$ According to the World Health Organization, the case fatality rate for COVID-19 patients with cancer as a comorbid condition was $7.6 \%$ vs. a case fatality rate of $3.8 \%$ in the entire COVID-19 population. Therefore, it becomes clear that cancer likely influences COVID-19 pathogenesis. More than half a million people in the United States are receiving chemotherapy and more than 1.5 million people will be diagnosed with cancer. Patients who are under active chemo/radiotherapy or recently underwent stem cell transplantation are particularly at higher risk. Despite of cancer patients being at high risk of developing severe complications following SARS-CoV-2 virus infection, data on COVID19 infection in patients with cancer is still limited. Immune dysregulation and chronic inflammation may be potential drivers of severe outcomes in COVID-19positive cancer patients. Therefore, it is important to study the underlying mechanisms that link COVID-19 and cancer. A better understanding of the mechanistic link between the two will help to prevent negative effects of infection and also enable the design of novel therapies that target cancer and COVID-19. It is also important to consider the problem of balancing chemotherapy and antiviral medication regimens and the timing of their administration. For some patients, chemotherapy must be postponed until completion of the antiviral course of therapy, while others cannot be subjected to the viral infection therapy when undergoing treatment for their cancer. ${ }^{3}$ One large study from Japan with evaluation of over 1000 breast cancer patients reported that chemotherapy for breast cancer patients with $\mathrm{HCV}$ infection is feasible, and according to their experience, viral load does not change during chemotherapy. ${ }^{39}$ Further elucidation is needed to clarify whether this outcome is cancer type or other factor-specific.

\section{Risks and Outcomes}

Some pressing questions that deserve attention include: Does SARS-CoV-2 infection cause or increase the risk of cancer? What is the relationship between tumor progression and COVID-19? Does SARS-CoV2 infection have an impact on the survival of cancer patients? Do COVID-19-positive cancer patients need to be treated differently? What is the biology of interconnection between COVID-19 and cancer?

There exists no clear evidence that SARS-CoV-2 is causative, related to, or modulates cancer pathobiology (Fig. 1). The transmission of SARS-CoV-2 is frequent in the community (infectivity index or R0 $\sim 2.5$ ) and often is the source of infection in immunocompromised cancer patients. With the limited amount of data available from patients, it is unclear whether all cancer patients are at a high risk of becoming infected with SARS-CoV-2 virus. However, it is clear that cancer patients have risk of becoming more sick from COVID-19 infection especially, those who are undergoing or have recently undergone cancer-associated treatments (chemotherapy, radiotherapy, stem cell transplantation or CAR-T cell therapy). ${ }^{33,44}$ With better availability of testing, it warrants consideration that cancer patients receiving anti-tumor treatments should have vigorous screening for COVID-19 infection and their immunosuppressive treatment regimens and dosages potentially decreased in the case of

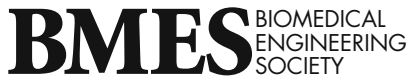




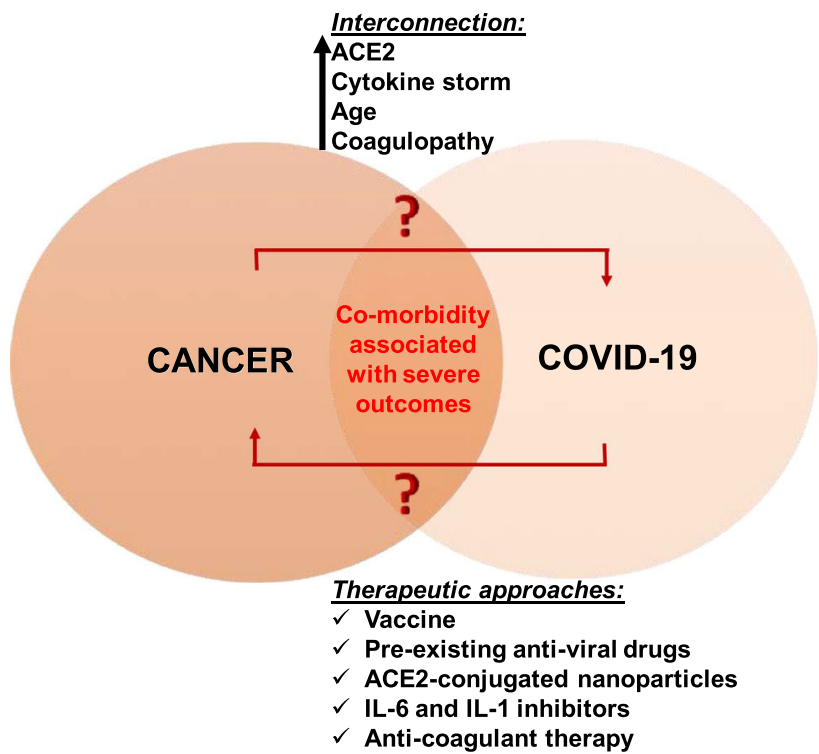

FIGURE 1. The interconnection between COVID-19 and cancer.

COVID-19 co-infection. ${ }^{59}$ Stronger personal protection facilities should be provided to cancer patients and more intensive observation should also be considered for COVID-19-positive cancer patients, older patients in particular. Additionally, unnecessary in-person clinic visits of cancer patients should be avoided to prevent the transmission of virus. This can be achieved through the use of oral therapies. Therefore, cancer patients are at risk for severity of infection but not for contracting the infection. Regarding the symptoms of COVID-19 in cancer patients, for the most part, the symptoms of COVID-19 are the same in cancer patients as in the general population (fever, coughing and shortness of breath). ${ }^{27,31}$ However, steroids or other treatments in cancer patients may suppress the fever symptom of COVID-19. If a cancer patient tests positive for COVID-19, the decision on treatment may depend on the type of cancer, stage of treatment, and severity of COVID-19.

With regards to the biological interconnection between COVID-19 and cancer, ACE2, cytokine storm, age and coagulopathy are few strong factors that connect COVID-19 and cancer. A deeper understanding of these connecting links may guide us in finding novel anti-viral and anti-cancer therapeutic options (Fig. 1).

\section{Angiotensin Converting Enzyme 2 ( ACE2)}

ACE2 is a carboxypeptidase that converts angiotensin I to angiotensin 1-9 and angiotensin II to angiotensin $1-7$. It is an important regulator of heart function and has a protective role in acute lung injury. ${ }^{12,50,51}$ Like SARS-CoV, SARS-CoV-2 also enters human cells through angiotensin 1 converting enzyme 2 (ACE2). ${ }^{56,60}$ The spike protein of SARS-CoV-2 virus binds to ACE2, and together with ACE2, enters the cell to replicate and expand. ${ }^{53}$ ACE2 is usually expressed in multiple organs, including respiratory, cardiovascular, digestive and urinary systems in healthy individuals, and its expression levels in specific cellular subtypes has been shown to be viral infectionand interferon-driven. ${ }^{11,21,62}$ In comparison to healthy individuals, the expression levels of ACE2 is different in cells and tissues of cancer patients. A recent study from China indicates that the expression of ACE2 is gradually increased from healthy control, adenoma, to colorectal cancer patients and speculates that cancer patients are more likely to be infected with SARSCoV-2 than healthy people. Lately, it has been reported that patients with tumors, with high expression level of ACE2, are more likely to be susceptible to SARS-CoV-2 infection and have poor prognosis. ${ }^{11,19,34}$ Renal tissue shows higher expression levels of ACE2, and this might explain why most COVID-19 patients have renal dysfunction. ${ }^{35,53}$ However, according to the Human Protein Atlas, higher levels of ACE2 in renal and liver cancers have been demonstrated to be associated with favorable prognosis. Furthermore, decreased levels of ACE2 were reported in non-small cell lung cancer (NSCLC), and its overexpression had a protective effect in NSCLC and breast cancer via inhibiting cell growth and angiogenesis. ${ }^{15,16,58}$ Moreover, ACE2 is present on the $\mathrm{X}$ chromosome, meaning males have one copy and females two, however the observed risk of infection is not lower in men compared to women. According to a study in 159 COVID-19 patients in Italy, male patients were reported to exhibit more severe outcomes than females. ${ }^{18}$ Reports suggest that ACE2 protects mice against acute lung injury and avian influenza. Some of the H5N1 infected patients that have higher ACE2 levels in their serum showed better outcomes to avian influenza infection, and treating mice with human ACE2 prevented lung injury. ${ }^{7,63}$ However, there is a possibility that the widespread distribution of ACE2 in multiple organs may be responsible for the fact that more deaths from COVID-19 have been caused by multiple organ dysfunction syndrome rather than respiratory failure. Unfortunately, insufficient research has been done to confirm the mechanistic link between ACE2 expression and SARS-CoV-2 infection in cancer. Thus, it would be worthwhile to test whether levels of ACE2 increases or decreases in various tissues of cancer patients and COVID-19 patients and how this impacts COVID-19 infection in these patients. 


\section{Cytokine Storm}

Components of both innate and adaptive immunity shape the course of malignancy. This also involves alterations in cytokine profiles in cancer patients. Cytokine storm or cytokine release syndrome (CRS) is a systemic inflammatory response that can be triggered by factors such as pathogenic infections, chimeric antigen receptor (CAR)-T cell therapy, antibody treatments and certain drugs. ${ }^{6,45}$ The induction of a cytokine storm is the root cause of pathogenic inflammation both in SARS-CoV and SARS-CoV-2 infection. Cao et al. reported the presence of a lethal cytokine storm in the immune system of severe SARSCoV-2 infected patients, with pneumonitis signatures in their lung CT scans. ${ }^{4}$ COVID-19 patients are reported to have elevated levels of interleukin-6 (IL-6), interleukin-1 beta (IL-1 $\beta$ ) in serum, tumor necrosis factor-alpha (TNF- $\alpha$ ), lactate dehydrogenase, and increased levels of circulating monocytes. ${ }^{5,54}$ These extensive pro-inflammatory and interferon antagonizing properties in COVID-19 patients mimic those observed in cancer patients receiving CAR-T cell therapy or immunotherapy. ${ }^{38}$ Though the cytokine levels in COVID-19 patients experiencing acute respiratory disease syndrome (ARDS) are lower than those observed in CRS in cancer patients receiving CAR-T cell or immunoglobulin therapies, the experience and understanding of cancer biologists and oncologists in modulating severe inflammatory response may prove highly beneficial in this setting. It is clear that cytokine release and macrophage activation results in the immunopathology in COVID-19 disease and thus, anti-inflammatory cancer drugs may be repurposed for decreasing the morbidity in COVID-19 patients.

$$
\text { Age }
$$

The risk of cancer increases as we grow older. According to the National Cancer Institute, a quarter of new cancer diagnoses are in individuals aged between 65 and 74 years of age. One possible explanation out of many include the fact that as we grow older we accumulate more mutations due to prolonged exposure to carcinogens. Other possible explanations include increased chronic inflammation in the microenvironment, weaker immune system and less effective DNA damage repair mechanisms. ${ }^{55}$ Similarly, older adults are at elevated risk for developing more serious complications from COVID-19 illness. According to the Center for Disease Control, 8 out of 10 COVID-19-related deaths in the USA have been in adults 65 years old and older. We may speculate that some of the underlying reasons for severe outcomes of SARS-CoV-2 virus infection in older individuals may be similar to those observed in cancer patients. However, further research must be done to identify the molecular interconnection between age, cancer and COVID-19.

\section{Coagulopathy}

Both, cancer and COVID-19 are associated with coagulopathy in a complex manner. Thrombotic and bleeding complications are leading causes of death in cancer patients and are often associated with the morbidities in COVID-19 patients. Various prothrombotic properties of tumor cells including release of cytokines, cysteine proteases, tumor microparticles and other pro-coagulants can cause an imbalance in hemostasis. Additionally, the contribution of pulmonary embolism in coagulopathy in cancer is also an active area of research. ${ }^{14}$ Disseminated intravascular coagulation (DIC) is associated with $71.4 \%$ of COVID-19 non-survivors and is a strong predictor of mortality in COVID-19 patients. Increased levels of Ddimer and prothrombin and decrease in fibrinogen was reported in these patients at days $10-14 .^{32,49,61}$ This emphasizes the importance of frequent and regular monitoring and maintaining of these factors in COVID-19 and cancer patients. More insights into the molecular connections of these disease conditions to coagulopathy may help in reducing the associated mortality in these patients.

\section{TREATMENT APPROACHES IN COVID-19 AND CANCER}

The success in treatment of viral infections and cancer is unprecedented, however the comorbidity of both conditions poses a major challenge in the management of these patients. The major points of consideration include the balancing and optimization of anti-cancer and anti-viral therapeutic regimens and the timeline of administration. For example, Borchardt et al. reported that for hepatitis $\mathrm{C}$ virus (HCV)-positive cancer patients, chemotherapy should be delayed until the course of anti-viral therapy and vice versa. ${ }^{3}$ On the contrary, after evaluation of over 1000 breast cancer patients in Japan it was observed that chemotherapy of $\mathrm{HCV}$-positive breast cancer patients is feasible with no significant adverse effects. ${ }^{39}$ However, when ovarian cancer patients undergoing chemotherapy receive influenza vaccine, they are unable to generate antibody response to the vaccination. ${ }^{8}$ It is worthwhile to examine whether both anti-cancer and anti-COVID-19 therapies can be administered simultaneously in a safe manner, and further efforts will be needed to address 
the degree to which such outcomes are dependent on the type of cancer, viral infection and therapy.

Clearly, the most promising approach for preventing or fighting a viral infection is via vaccination. However, the vaccine development against SARSCoV-2 virus remains in its early stages and so far, no approved vaccine candidate exists. ${ }^{42}$ Globally, pharmaceutical and research organizations are aggressively pursuing efforts to develop an effective candidate vaccine to neutralize SARS-CoV-2 virus and are also directing preexisting anti-viral drugs as well as convalescent serum from COVID-19 recovered patients. However, in the meantime options are needed to support the severe inflammatory response and respiratory complications that occur in severe COVID-19 patients. Cocktails of different monoclonal antibodies (mAbs) that recognize the different epitopes on the viral surface may have better efficacy in neutralizing the SARSCoV-2 virus. Cytokines present another promising target, especially IL-6 as higher levels are correlated with cytokine storm in COVID-19 patients. Oncologists have been using IL-6 inhibitors (for example, tocilizumab and siltuximab mAbs) for the management of CRS in cancer patients receiving CAR-T cell therapy. Another pro-inflammatory cytokine upstream of IL-6 is IL-1, which is also upregulated in CRS. IL-1 receptor antagonists such as anakinra have been frequently used to treat arthritis patients with CRS symptoms. Calcineurin inhibitors present another class of nontoxic immunosuppressants that impair $\mathrm{T}$ cell function and thereby reduce cytokine levels. These may help to mitigate the severe COVID-19 symptoms in patients. Lung cancer patients undergoing immunotherapy and with immune related severe adverse responses have benefitted from tocilizumab. ${ }^{48} \mathrm{It}$ is important to consider however that comorbidities from CRS symptoms due to cancer immunotherapies and SARS-CoV-2 infection could be fatal in patients.

The presence of viral gene components vital for the unchecked proliferation of viruses in host cancer cells may also provide targets for directed and tolerable effective therapies. ${ }^{26,28}$ This may also help researchers to understand key differences between the biology of COVID-19-infected cancer vs. normal host cells. Increased understanding of the relevant constituents of the immune system is likely to lead to new biological strategies to fight the serious co-morbidity arising from viral infections in cancer patients. Recent insights into the roles of dendritic cells, $\mathrm{T}$ cells and natural killer cells in the pathology and therapies of cancer and viral infections motivate one to better understand and develop novel immunomodulatory therapeutic strategies to co-target these diseases. As innate effectors, functional natural killer cells can orchestrate antiviral responses against influenza infection and they are also reported as potential host-directed anti-cancer agents. ${ }^{13,41,52}$ Moreover, due to their associated negligible graft vs. host signature, they may provide a safer and faster alternative to co-target COVID-19 and cancer. ${ }^{2}$ However, further evidence is needed to confirm the therapeutic benefit of these immunotherapies in COVID-19 patients. Care must be taken to avoid cytokine storm effects in COVID-19 patients following these immunomodulatory therapies. By increasing the bioavailability and effectiveness of the payload, nanoparticles provide a safer delivery option for various anti-viral drugs including vaccine candidates. ${ }^{47}$ Nanoparticles decorated with recombinant human ACE2 protein on their surface may provide an effective therapeutic option for COVID-19 patients. ACE2conjugated nanoparticles will bind to the spike protein of SARS-CoV-2 virus and may thus neutralize the virus and prevent it from binding the ACE2 receptor present on host cells.

\section{FUTURE RESEARCH PERSPECTIVES}

Studies are needed to understand how a normal cell vs. cancerous cell interacts with the SARS-CoV-2 virus. This will provide knowledge on which cells are most susceptible to SARS-CoV-2, and how exactly we can target SARS-CoV-2 virus with immunotherapies, drugs and vaccines to prevent or treat COVID-19 and cancer. To study the comparative pathogenesis of COVID-19, one needs to understand and establish suitable animal models that recapitulate the comorbidity of various types of cancers and COVID-19 in patients with accuracy. Likewise, similar models should be used for testing of COVID-19 post-exposure therapeutics. Additionally, the role of specific SARSCoV-2 proteins may be dissected by creating chimeric mouse models that express SARS-CoV-2 proteins in some tissues. Ziegler et al. recently demonstrated the specific human cells that SARS-CoV-2 primarily targets for infection and that human ACE2 expression in epithelial cells is interferon dependent. ${ }^{62}$ This indicates that any potential use of interferon as a treatment to fight COVID-19 will require careful monitoring to determine if and when it might help patients. Notably, a significantly weaker induction of murine ACE2 was observed in response to interferon or viral infection. ${ }^{62}$ This must be accounted for when establishing murine models for carrying out COVID-19 related research. Therefore, humanized models that are permissive to SARS-CoV-2 and capable of harboring human tumors will faithfully mimic the human condition. Ultimately, these models may be utilized to develop cancer therapies that exploit SARS-CoV-2 for cancer therapeutics. Identifying the relevant components of immune system 
will likely lead to new biological strategies to fight the serious co-morbidity arising from viral infections in cancer patients. It would be interesting to determine whether active SARS-CoV-2 virus can lead to cancer in mice and reveal the pathogenic impact of SARSCoV-2 infection in established cancer models.

In summary, further research is needed to improve the understanding of the biology of SARS-CoV-2 virus, its molecular connection to cancer, and the temporal features of SARS-CoV-2-induced inflammatory response in relation to the timing of therapeutic interventions in general, and more importantly, in cancer patients. It is likewise important for cancer patients to maintain a healthy lifestyle and take steps to support their immune system.

\section{FUNDING}

This work was supported by National Institute of Health Grant No. R01 CA203991 to M.R.K.

\section{AUTHOR CONTRIBUTIONS}

N.J. and M.R.K. wrote the manuscript.

\section{CONFLICT OF INTEREST}

The authors N.J. and M.R.K. declare that they have no conflicts of interest.

\section{REFERENCES}

${ }^{1}$ Alqahtani, F. Y., et al. Prevalence of comorbidities in cases of Middle East respiratory syndrome coronavirus: a retrospective study. Epidemiol. Infect. 2018. https://doi.org/ $10.1017 / \mathrm{s} 0950268818002923$.

${ }^{2}$ Benjamin, J. E., S. Gill, and R. S. Negrin. Biology and clinical effects of natural killer cells in allogeneic transplantation. Curr. Opin. Oncol. 22(2):130-137, 2010.

${ }^{3}$ Borchardt, R. A., and H. A. Torres. Challenges in managing hepatitis $\mathrm{C}$ virus infection in cancer patients. World J. Gastroenterol. 20(11):2771-2776, 2014.

${ }^{4} \mathrm{Cao}$, J., et al. Clinical features and short-term outcomes of 102 patients with corona virus disease 2019 in Wuhan, China. Clin. Infect. Dis. 2020. https://doi.org/10.1093/cid/c iaa243.

${ }^{5}$ Cao, J., et al. Clinical features and short-term outcomes of 18 patients with corona virus disease 2019 in intensive care unit. Intensive Care Med. 2020. https://doi.org/10.1056/N EJMoa2001017.

${ }^{6}$ Chatenoud, L., et al. In vivo cell activation following OKT3 administration. Systemic cytokine release and modulation by corticosteroids. Transplantation 49(4):697702, 1990.
${ }^{7}$ Chen, L., and G. Hao. The role of angiotensin-converting enzyme 2 in coronaviruses/influenza viruses and cardiovascular disease. Cardiovasc. Res. 2020. https://doi.org/10. 1093/cvr/cvaa093.

${ }^{8} \mathrm{Chu}, \quad$ C. S., et al. Immunologic consequences of chemotherapy for ovarian cancer: impaired responses to the influenza vaccine. Vaccine 31(46):5435-5442, 2013.

${ }^{9}$ Cooksley, C. D., et al. Epidemiology and outcomes of serious influenza-related infections in the cancer population. Cancer 104(3):618-628, 2005.

${ }^{10}$ Coussens, L. M., and Z. Werb. Inflammation and cancer. Nature 420(6917):860-867, 2002.

${ }^{11}$ Dai, Y. J., et al. A profiling analysis on the receptor ACE2 expression reveals the potential risk of different type of cancers vulnerable to SARS-CoV-2 infection. Ann. Transl. Med. 8(7):481, 2020.

${ }^{12}$ Donoghue, M., et al. A novel angiotensin-converting enzyme-related carboxypeptidase (ACE2) converts angiotensin I to angiotensin 1-9. Circ. Res. 87(5):E1-E9, 2000.

${ }^{13}$ Eyileten, C., et al. Immune cells in cancer therapy and drug delivery. Mediators Inflamm. 2016. https://doi.org/10.1155/ 2016/5230219.

${ }^{14}$ Falanga, A., M. Marchetti, and A. Vignoli. Coagulation and cancer: biological and clinical aspects. J. Thromb. Haemost. 11(2):223-233, 2013.

${ }^{15}$ Feng, Y., et al. The angiotensin-converting enzyme 2 in tumor growth and tumor-associated angiogenesis in nonsmall cell lung cancer. Oncol. Rep. 23(4):941-948, 2010.

${ }^{16}$ Feng, Y., et al. Overexpression of ACE2 produces antitumor effects via inhibition of angiogenesis and tumor cell invasion in vivo and in vitro. Oncol. Rep. 26(5):1157-1164, 2011.

${ }^{17}$ Gao, L., et al. Tumor-derived exosomes antagonize innate antiviral immunity. Nat. Immunol. 19(3):233-245, 2018.

${ }^{18}$ Grasselli, G., et al. Baseline characteristics and outcomes of 1591 patients infected with SARS-CoV-2 admitted to ICUs of the Lombardy Region, Italy. JAMA 2020. https://doi. org/10.1001/jama.2020.5394.

${ }^{19}$ Guan, W. J., et al. Comorbidity and its impact on 1590 patients with COVID-19 in China: a nationwide analysis. Eur. Respir. J. 2020. https://doi.org/10.1183/13993003.005 47-2020.

${ }^{20}$ Habel, L. A., et al. The epidemiology of herpes zoster in patients with newly diagnosed cancer. Cancer Epidemiol. Biomarkers Prev. 22(1):82-90, 2013.

${ }^{21}$ Hamming, I., et al. Tissue distribution of ACE2 protein, the functional receptor for SARS coronavirus. A first step in understanding SARS pathogenesis. J. Pathol. 203(2):631-637, 2004.

${ }^{22}$ Hanna, T. P., G. A. Evans, and C. M. Booth. Cancer, COVID-19 and the precautionary principle: prioritizing treatment during a global pandemic. Nat. Rev. Clin. Oncol. 17(5):268-270, 2020.

${ }^{23}$ Hotchkiss, R. S., and L. L. Moldawer. Parallels between cancer and infectious disease. N. Engl. J. Med. 371(4):380383, 2014.

${ }^{24}$ Iglar, K., A. Kopp, and R. H. Glazier. Herpes zoster as a marker of underlying malignancy. Open Med. 7(2):e68-e73, 2013.

${ }^{25}$ Iheagwara, U. K., et al. Influenza virus infection elicits protective antibodies and $\mathrm{T}$ cells specific for host cell antigens also expressed as tumor-associated antigens: a new view of cancer immunosurveillance. Cancer Immunol. Res. 2(3):263-273, 2014. 
${ }^{26}$ Johnson, M. E., P. G. Cantalupo, and J. M. Pipas. Identification of head and neck cancer subtypes based on human papillomavirus presence and E2F-regulated gene expression. mSphere 2018. https://doi.org/10.1128/mspher e.00580-17.

${ }^{27}$ Kalinsky, K., et al. Characteristics and outcomes of patients with breast cancer diagnosed with SARS-Cov-2 infection at an academic center in New York City. Breast Cancer Res. Treat. 2020. https://doi.org/10.1007/s10549-0 20-05667-6.

${ }^{28}$ Kim, Y., C. M. Pierce, and L. A. Robinson. Impact of viral presence in tumor on gene expression in non-small cell lung cancer. BMC Cancer 18(1):843, 2018.

${ }^{29}$ Kohler, M., et al. Enhanced tumor susceptibility of immunocompetent mice infected with lymphocytic choriomeningitis virus. Cancer Immunol. Immunother. 32(2):117124, 1990.

${ }^{30}$ Kohlhapp, F. J., et al. Non-oncogenic acute viral infections disrupt anti-cancer responses and lead to accelerated cancer-specific host death. Cell Rep. 17(4):957-965, 2016.

${ }^{31}$ Leonetti, A., et al. COVID-19 in lung cancer patients receiving ALK/ROS1 inhibitors. Eur. J. Cancer 132:122124, 2020

${ }^{32}$ Levi, M., et al. Coagulation abnormalities and thrombosis in patients with COVID-19. Lancet Haematol. 2020. http s://doi.org/10.1016/S2352-3026(20)30145-9.

${ }^{33}$ Liang, W. H., et al. Clinical characteristics and outcomes of hospitalised patients with COVID-19 treated in Hubei (epicenter) and outside Hubei (non-epicenter): a nationwide analysis of China. Eur. Respir. J. 2020. https://doi.org/10. 1183/13993003.00562-2020.

${ }^{34}$ Liang, W., et al. Cancer patients in SARS-CoV-2 infection: a nationwide analysis in China. Lancet Oncol. 21(3):335337, 2020.

${ }^{35} \mathrm{Lu}$, R., et al. Genomic characterisation and epidemiology of 2019 novel coronavirus: implications for virus origins and receptor binding. Lancet 395(10224):565-574, 2020.

${ }^{36}$ Martinez, A., et al. Risk factors associated with severe outcomes in adult hospitalized patients according to influenza type and subtype. PLoS ONE 14(1):e0210353, 2019.

${ }^{37}$ Mauskopf, J., et al. The burden of influenza complications in different high-risk groups: a targeted literature review. $J$. Med. Econ. 16(2):264-277, 2013.

${ }^{38}$ Mehta, P., et al. COVID-19: consider cytokine storm syndromes and immunosuppression. Lancet 395(10229):10331034, 2020.

${ }^{39}$ Miura, Y., et al. The safety of chemotherapy for breast cancer patients with hepatitis $\mathrm{C}$ virus infection. $J$ Cancer 4(6):519-523, 2013.

${ }^{40}$ Newman, J. H., and A. Zloza. Infection: a cause of and cure for cancer. Curr. Pharmacol. Rep. 3(6):315-320, 2017.

${ }^{41}$ Papaioannou, N. E., et al. Harnessing the immune system to improve cancer therapy. Ann. Transl. Med. 4(14):261, 2016.

${ }^{42}$ Pinto, D., et al. Cross-neutralization of SARS-CoV-2 by a human monoclonal SARS-CoV antibody. Nature 2020. h ttps://doi.org/10.1038/s41586-020-2349-y.

${ }^{43}$ Rahmatika, D., et al. Inhibitory effects of viral infection on cancer development. Virology 528:48-53, 2019.

${ }^{44}$ Sahu, K. K., et al. Facing COVID-19 in the hematopoietic cell transplant setting: a new challenge for transplantation physicians. Blood Cells Mol. Dis. 83:102439, 2020.

${ }^{45}$ Shimabukuro-Vornhagen, A., et al. Cytokine release syndrome. J. Immunother. Cancer 6(1):56, 2018.
${ }^{46}$ Sigel, K., et al. Cancer screening in patients infected with HIV. Curr. HIV/AIDS Rep. 8(3):142-152, 2011.

${ }^{47}$ Singh, L., et al. The role of nanotechnology in the treatment of viral infections. Ther. Adv. Infect. Dis. 4(4):105131,2017

${ }^{48}$ Stroud, C. R., et al. Tocilizumab for the management of immune mediated adverse events secondary to PD-1 blockade. J. Oncol. Pharm. Pract. 25(3):551-557, 2019.

${ }^{49}$ Tang, N., et al. Abnormal coagulation parameters are associated with poor prognosis in patients with novel coronavirus pneumonia. J. Thromb. Haemost. 18(4):844 847,2020

${ }^{50}$ Tipnis, S. R., et al. A human homolog of angiotensinconverting enzyme. Cloning and functional expression as a captopril-insensitive carboxypeptidase. J. Biol. Chem. 275(43):33238-33243, 2000.

${ }^{51}$ Vickers, C., et al. Hydrolysis of biological peptides by human angiotensin-converting enzyme-related carboxypeptidase. J. Biol. Chem. 277(17):14838-14843, 2002.

${ }^{52}$ Waggoner, S. N., et al. Roles of natural killer cells in antiviral immunity. Curr. Opin. Virol. 16:15-23, 2016.

${ }^{53}$ Wan, Y., et al. Receptor recognition by the novel coronavirus from wuhan: an analysis based on decade-long structural studies of SARS coronavirus. J. Virol. 2020. h ttps://doi.org/10.1128/JVI.00127-20.

${ }^{54}$ Wen, W., et al. Immune cell profiling of COVID-19 patients in the recovery stage by single-cell sequencing. Cell Discov. 6:31, 2020.

${ }^{55}$ White, M. C., et al. Age and cancer risk: a potentially modifiable relationship. Am. J. Prev. Med. $46(3 \mathrm{Suppl}$ 1):S7-S15, 2014.

${ }^{56} \mathrm{Xu}, \mathrm{H}$., et al. High expression of ACE2 receptor of 2019$\mathrm{nCoV}$ on the epithelial cells of oral mucosa. Int. J. Oral Sci. 12(1):8, 2020.

${ }^{57}$ Yan, L., Q. Cai, and Y. Xu. The ubiquitin-CXCR4 axis plays an important role in acute lung infection-enhanced lung tumor metastasis. Clin. Cancer Res. 19(17):4706-4716, 2013.

${ }^{58}$ Zhang, Q., et al. ACE2 inhibits breast cancer angiogenesis via suppressing the VEGFa/VEGFR2/ERK pathway. $J$. Exp. Clin. Cancer Res. 38(1):173, 2019.

${ }^{59}$ Zhang, L., et al. Clinical characteristics of COVID-19-infected cancer patients: a retrospective case study in three hospitals within Wuhan, China. Ann. Oncol. 31:894-901, 2020.

${ }^{60}$ Zhou, P., et al. A pneumonia outbreak associated with a new coronavirus of probable bat origin. Nature 579(7798):270-273, 2020.

${ }^{61}$ Zhou, F., et al. Clinical course and risk factors for mortality of adult inpatients with COVID-19 in Wuhan, China: a retrospective cohort study. Lancet 395(10229):1054-1062, 2020.

${ }^{62}$ Ziegler, C. G. K., et al. SARS-CoV-2 Receptor ACE2 is an interferon-stimulated gene in human airway epithelial cells and is detected in specific cell subsets across tissues. Cell 2020. https://doi.org/10.1016/j.cell.2020.04.035.

${ }^{63} \mathrm{Zou}, \mathrm{Z}$., et al. Angiotensin-converting enzyme 2 protects from lethal avian influenza A $\mathrm{H} 5 \mathrm{~N} 1$ infections. Nat. Commun. 5:3594, 2014.

Publisher's Note Springer Nature remains neutral with regard to jurisdictional claims in published maps and institutional affiliations. 